\title{
Comparação dos níveis pressóricos de um grupo de hipertensos submetidos a um protocolo de orientações e de outro, sem
}

\section{Comparison of blood pressure levels of hypertensive patients submitted to a protocol of conduct and other without guidance}

\author{
Edinéia Reis Castelo Bonalume ${ }^{1}$; Vanessa Delgado de Abreu²; Zaide Silva Frazão³ \\ Graduanda de Enfermagem - Uninove \\ Graduanda de Enfermagem - Uninove \\ Mestre em Reabilitação/Enfermagem - Unifesp \\ Docente Saúde do Adulto - Uninove \\ Endereço para correspondência \\ Vanessa Delgado de Abreu \\ Praça N. S. da Anunciação, 80 AP. 55-2 \\ 03542-120 Vila Guilhermina, São Paulo - SP \\ delgado_vanessa@hotmail.com
}

\begin{abstract}
Resumo
Busca-se, por meio deste estudo, comparar os níveis pressóricos de grupos controle e experimental na atenção primária pela aplicação de um instrumento não farmacológico. Trata-se de Estudo de Campo, de caráter observacional, randomizado, qualitativo, fundamentado na verificação pressórica diária de uma amostra $\mathrm{n}=20$, distribuída em dois grupos: o controle $\mathrm{n}=10$ e o experimental $\mathrm{n}=10$, em que foram implementadas intervenções com base na mudança de estilo de vida por meio de uma alimentação saudável, restrição a sódio, abandono de hábitos tabagistas e atividades físicas aeróbicas sob orientação. Esses dados foram registrados em gráficos e discutidos. A amostra correspondia a 30\% do sexo masculino e $70 \%$ do feminino, idade mediana de 56 anos, $100 \%$ hipertensos e maiores, $70 \%$ farmacodependentes, $30 \%$ praticavam esportes, $60 \%$ estavam em sobrepeso, $50 \%$ faziam dieta alimentar, $20 \%$ etilistas, 10\% tabagistas, $90 \%$ diabéticos e $40 \%$ dislipidêmicos. A pesquisa aponta as estratégias para a prevenção da HAS, com grande importância especialmente no âmbito da atenção primária, produzindo consideráveis impactos socioeconômicos.
\end{abstract}

Descritores: Hipertensão; Prevenção; Protocolo

\begin{abstract}
It is intended, in this study, to compare the levels of blood pressure in experimental and control groups in primary care through the application of a nonpharmacological instrument. It is a field study of randomized, observational, qualitative nature, based on check of daily pressure of a sample $n=20$, distributed in two groups: control group $n=10$ and $n=10$ experimental group, in which intervention was implemented based on the change of lifestyle including healthy nutrition, sodium restriction, cessation of smoking and aerobic physical activity under supervision. The data were recorded in graphs and discussed. The sample comprised $30 \%$ of males and $70 \%$ of females, median age of 56 years, hypertension and increased $100 \%, 70 \%$ drug addicted, $30 \%$ practiced sports, $60 \%$ were overweight, $50 \%$ were diet, $20 \%$ alcohol users, $10 \%$ smokers, $90 \%$ diabetics and $40 \%$ dyslipidemic. The research outlines the strategies for the prevention of hypertension with particular importance in the context of primary care producing considerable socioeconomic impacts.
\end{abstract}

Key words: Hypertension; Prevention; Protocol. 


\section{Introdução}

O impacto das doenças crônicas não transmissíveis (DCNT) na saúde das populações é crescente em todo o mundo. Em 2005, aproximadamente 35 milhões de pessoas morreram por esse motivo, sendo $80 \%$ desses óbitos em países de média e baixa renda. A hipertensão arterial sistêmica (HAS) configura-se como um dos agravos crônicos mais comuns e com repercussões clínicas mais graves. Estima-se que, em todo o mundo, 7,1 milhões de pessoas morram anualmente por causa da pressão sanguínea elevada e que $4,5 \%$ da carga de doença no mundo seja causada pela $\mathrm{HAS}^{1}$. Estudos epidemiológicos demonstram que um em cada cinco indivíduos com idade superior a 18 anos, no mundo, apresenta hipertensão arterial $^{2}$.

A hipertensão arterial tem elevada prevalência no Brasil e constitui um dos maiores desafios para os profissionais de saúde, pois é decorrente do novo perfil populacional de envelhecimento progressivo e, consequentemente, da maior exposição aos fatores de risco cardiovasculares, o que aumenta a importância de ações preventivas e educacionais que melhorem os hábitos e a qualidade de vida da clientela ${ }^{3}$.

A hipertensão arterial sistêmica (HAS) é uma síndrome de origem multifatorial, caracterizada pelo aumento das cifras pressóricas arteriais, ou seja, a partir de $135 \mathrm{mmhg}$ para a Pressão Arterial Sistólica (PAS) e de 85 mmhg para a Pressão Arterial Diastólica (PAD), o que ocasiona anormalidades cardiovasculares e metabólicas que podem levar a alterações funcionais e/ou estruturais de vários órgãos, principalmente coração, cérebro, rins e vasos periféricos ${ }^{4}$.

Entre os fatores de risco para a mortalidade cardiovascular, a hipertensão arterial é responsável por $40 \%$ das mortes por acidente vascular, e $25 \%$, por doença arterial coronariana ${ }^{2}$.

Os resultados de estatísticas recentes evidenciam que, dependendo da região do Brasil, de $22 \%$ a $44 \%$ da população urbana adulta é portadora de hipertensão arterial. Esses números são extremamente importantes, uma vez que a hipertensão arterial está diretamente relacionada a eventos cerebrovasculares, coronariopatia e mortalidade ${ }^{5}$.

Os estudos têm demonstrado consistentemente que o risco decorrente das elevações da pressão arterial tanto sistólica quanto diastólica é crescente e contínuo, mesmo para valores ainda não considerados como de hipertensão ${ }^{6}$.

Na concepção de muitos estudiosos, as dificuldades de controle da HA estão relacionadas a algumas peculiaridades da doença de caráter assintomático, evolução lenta e marcada pela cronicidade, que fazem com que não seja considerada doença ou algo que precise ser cuidado. Assim, os portadores não sentem necessidade de modificar os hábitos relacionados ao trabalho, ao meio social e à dinâmica familiar, até que surjam as primeiras complicações provocadas pela doença ${ }^{7}$.

O diagnóstico precoce reduz o risco de complicação. Nesse contexto, as ações preventivas em atenção primária justificam sua implementação, sobretudo ao considerar que $60 \%$ a $80 \%$ dos casos de HA podem ser detectados e tratados na rede básica ${ }^{8}$.

A constante análise e o levantamento de informações sobre esse agravo são de fundamental importância para planejadores e gestores de saúde ${ }^{1}$.

A vivência da educação em saúde por meio de grupos favorece a participação como forma de garantir ao indivíduo e à comunidade a possibilidade de decidir sobre seus próprios destinos, e a capacitação desses sujeitos para atuar na melhoria do seu nível de saúde ${ }^{4}$.

A hipertensão arterial, mesmo que reconhecida como problema relevante, ainda é tratada e controlada inadequadamente. Embora os avanços científicos e tecnológicos nas últimas décadas venham facilitando a identificação dos fatores de risco, o diagnóstico precoce e seus agravos, o emprego de nova terapêutica medicamentosa e as ações educativas para as mudanças no estilo de vida, promovidas por diferentes meios de comunicação, em que se destaca a atuação dos profissionais de saúde, em especial a 
dos da rede básica, em todos os municípios brasileiros, ainda não são os ideais ${ }^{7}$.

Grande ênfase tem-se dado às medidas não farmacológicas, relacionadas à mudança no estilo de vida, para prevenção e controle dos níveis pressóricos elevados, que devem ser adotadas por todos os portadores de hipertensão, inclusive pelos farmacodependentes, e por pessoas com antecedência familiar de doença hipertensiva9.

Apesar de o seu surgimento estar intimamente relacionado aos fatores de risco constitucionais, idade, sexo, raça/cor e história familiar, a prevenção ou postergação da HAS pode ser obtida pela eliminação ou controle dos fatores de risco ambientais, sedentarismo, sobrepeso/obesidade, consumo de alimentos insalubres (excesso de sal, gordura animal, preferência por carboidratos simples e complexos, ingestão diária acima de 100 $\mathrm{ml}$ de café ou de bebidas que contêm cafeína, uso abusivo de álcool, estresse não gerenciado e tabagismo), adotando-se estilo de vida saudável que, por sua vez, inclui a prática regular de exercício físico, o controle de peso, a alimentação saudável, o consumo moderado de álcool, a abolição do hábito do tabagismo e o gerenciamento do estresse ${ }^{4}$. Diversos estudos relatam que o efeito do exercício físico nos níveis pressóricos é tão expressivo que uma única sessão de exercício físico aeróbio provoca reduções na pressão arterial por várias horas, após sua realização. Além disso, quando realizado constantemente, o exercício físico aeróbio é capaz de prevenir e tratar a hipertensão arterial ${ }^{2}$.

Contudo, apenas parte dos portadores que mantêm valores satisfatórios da pressão arterial (PA<135 / 85 mmhg) usa a medicação de forma correta e adere às medidas terapêuticas, especialmente, as que envolvem mudanças de hábitos alimentares, abandono de vícios, tais como tabagismo e alcoolismo, e a incorporação de atividades físicas. Com isso, a prevenção e o tratamento da doença passam a representar desafios às autoridades sanitárias, aos profissionais de saúde, aos portadores e familiares envolvidos na promoção de saúde ${ }^{7}$.
A relevância da hipertensão arterial como importante fator de risco cardiovascular, sua alta prevalência mundial e o aumento da probabilidade de desfechos circulatórios fatais ou não fatais, quando a ela estão associados outros fatores de risco, tornam muito importante o conhecimento de sua ocorrência nacional e regional, assim como a correlação com outros possíveis fatores potencialmente desencadeantes de eventos cardiocirculatórios ${ }^{10}$.

Com base no exposto, considera-se a aplicação de um instrumento para o controle da HAS um grande desafio e compromisso. A validação de tal mecanismo no âmbito da atenção primária favorece a população local, criando subsídios para tratá-la e melhorando suas possibilidades de saúde cardiovascular e a qualidade de vida e de educação em saúde.

\section{Objetivos}

Comparar os níveis pressóricos de grupos controle e experimental na atenção primária pela aplicação de um instrumento não farmacológico.

\section{Materiais e métodos}

\section{Tipo de estudo}

Estudo de campo, de caráter observacional randomizado e qualitativo.

\section{População e amostra}

Realizou-se uma pesquisa de campo com a população da $\mathrm{COHAB} \mathrm{I,} \mathrm{pertencente} \mathrm{à} \mathrm{sub-}$ prefeitura da Penha. O conjunto habitacional abrange uma área de 375.419,19 $\mathrm{m}^{2}$ e uma estimativa populacional de aproximadamente 35.950 habitantes. Foi selecionada uma amostra constituída de 20 pessoas que, após assinarem o consentimento livre e esclarecido de acordo com a resolução 196/96 do Conselho Nacional de Saúde, submeteram-se a um instrumen- 
to para o controle da hipertensão arterial. Foi aplicado um questionário para avaliar os critérios de inclusão e de exclusão para participar da pesquisa. Os critérios de inclusão foram os seguintes:

- Faixa etária de 18 a 100 anos;

- Sexo feminino e/ou masculino;

- HAS de base;

- Farmacodependentes;

- Sedentários;

- Etilistas;

- Tabagistas;

- Abstinentes do álcool e tabaco;

- Comorbidades associadas: DM e dislipidemias;

- Obesidade;

- Antecedentes familiares de HAS;

- Doenças crônicas não transmissíveis;

- Deficientes físicos.

Os critérios de exclusão foram os seguintes:

- Menores;

- Usuários de drogas ilícitas;

- Deficientes mentais;

- Gestantes.

Portanto, considerou-se apto a colaborar com a pesquisa o indivíduo maior do sexo feminino ou masculino, heterossexual, homossexual, bissexual ou transexual, com história de hipertensão arterial leve, moderada ou severa, que usa medicações para seu controle como fármacos anti-hipertensivos de quaisquer classes, os sedentários em que se incluíam indivíduos que não realizavam atividades físicas, etilistas formados por consumidores de teores alcoólicos social ou cronicamente, tabagistas que consumiam qualquer quantidade de produtos fumígeros e também abstinentes ao álcool e tabaco, ou pessoas que apresentassem comorbidades associadas ao diabetes e dislipidemias, ainda obesos ou com antecedentes familiares de pressão alta e com histórico de doenças crônicas não transmissíveis, ou fossem deficientes físicos.
Dois critérios foram fundamentais e obrigatórios para que o indivíduo fosse inserido no grupo de pesquisa: a condição de maior e hipertenso. Os demais critérios de inclusão apenas nos ajudaram a qualificar com maior propriedade o grupo pesquisado. $\mathrm{O}$ colaborador que foi classificado com qualquer um dos itens referidos no critério de exclusão foi considerado inapto à pesquisa.

\section{Local da pesquisa}

A pesquisa foi desenvolvida na UBS Padre Manoel da Nóbrega, situada a Av. Padre Francisco de Toledo, 545, na cidade de São Paulo.

\section{Coleta de dados}

Os dados da pesquisa foram coletados entre 14/1/2009 e 15/2/2009 após a autorização do Comitê de Ética e Pesquisa CEP/SMS; em segui$\mathrm{da}$, selecionados candidatos concordantes com as condições preestabelecidas para a inclusão no estudo. Teve início a divisão dos participantes em dois grupos independentes que continham, obrigatoriamente, 10 integrantes cada. A população constituiu-se de pessoas hipertensas que correspondiam à livre demanda da UBS. A escolha para os grupos foi de origem aleatória. $\mathrm{O}$ grupo total constituído de 20 participantes foi denominado Mitologia Grega, e sua subdivisão, Zeus e Júpiter, cada qual com 10 integrantes. Participaram do grupo denominado Zeus 10 integrantes codificados em Poseidon, Hera, Hades, Palas Atena, Apolo, Ártemis, Afrodite, Hermes, Ares e Hefesto. E o Júpiter, constituído por Netuno, Juno, Plutão, Minerva, Febo, Diana, Vênus, Mercúrio, Marte e Vulcano, foi denominado como grupo experimental. Para esse grupo, aplicou-se o protocolo de condutas especificado que busca melhorar o estilo de vida por meio da mudança de comportamento que ocorreria pela alimentação saudável, por meio da redução de sal, e pelo combate ao tabagismo. Para controle da HAS, aos pacientes dos grupos Zeus, controle e cego, que se submeteram a medidas 
pressóricas sem nenhum instrumento de orientação, foram propostas a abstinência ou redução de $50 \%$ do consumo e atividade física aeróbica -caminhadas de 30min/dia, no mínimo, três vezes por semana. O procedimento foi realizado após dez minutos da chegada do colaborador a UBS e do seu respectivo repouso nesse período. Decorrido esse tempo, o paciente era posicionado em uma cadeira e orientado a descruzar as pernas, mantendo os pés apoiados no chão e o dorso recostado na cadeira, de forma que ficasse relaxado. Seu braço esquerdo era posicionado na altura do coração aproximadamente ao $4^{\circ}$ EICE, apoiado com a palma da mão voltada para cima. As roupas do braço eram removidas, permitindo a colocação do manguito sem interferências. Para escolha do manguito, foi realizada previamente a medição da circunferência braquial, observando-se as recomendações da American Heart Association ${ }^{11}$, e, em seguida, posicionado sem folgas de 2 a $3 \mathrm{~cm}$ acima da fossa cubital. Foi empregado o método palpatório e, após um minuto, sequencialmente, o método auscultatório. Para o procedimento, utilizou-se esfigmomanômetro de coluna de mercúrio disponível na UBS. O paciente era orientado a não falar durante a medição. Seu comparecimento a UBS para medição pressórica era registrado por meio da folha de frequência diária, perfazendo um total de 15 dias úteis subsequentes.

\section{Pressupostos éticos}

Este estudo preconizou os preceitos éticos e legais que norteiam a utilização dos seres humanos em pesquisas. Para tanto, foi utilizada como base a resolução 196/96 do Conselho Nacional de Saúde, que orienta e viabiliza o norteamento científico para esses casos.

Inicialmente, o projeto foi encaminhado ao Comitê de Ética em Pesquisas da SMS e, após parecer favorável, foram iniciados os procedimentos para seu desenvolvimento.

Após a seleção dos colaboradores para a pesquisa de campo, os escolhidos foram devidamente informados, de forma clara e compreensí- vel, acerca de todos os procedimentos, da justificativa e objetivos do estudo.

A assinatura do Termo de Livre Consentimento Esclarecido integrou oficialmente o colaborador ao estudo. No entanto, foi enfaticamente mencionada a condição de livre-arbítrio que lhe permite desistir da pesquisa mesmo depois de assinado o termo.

Ficam estabelecidos o anonimato e a utilização de codinomes já mencionados neste documento como forma de preservar a imagem dos colaboradores, todavia os demais dados serão divulgados.

\section{Resultados}

Participaram do estudo 20 indivíduos distribuídos em dois grupos: o experimental $(n=10)$ e o controle $(n=10)$. Indivíduos pertencentes ao grupo experimental submeteram-se a um protocolo de condutas em relação a Hipertensão Arterial Sistêmica (HAS), enquanto os do grupo controle também foram avaliadas no mesmo período, porém sem orientações.

$\mathrm{Na}$ amostra analisada, composta de 10 indivíduos representantes do grupo experimental, $30 \%$ correspondiam ao sexo masculino, e $70 \%$, ao feminino. A idade média e a mediana eram de 56 anos e não houve idade moda. Dos colaboradores questionados, $100 \%$ eram maiores e hipertensos. Desse total, $90 \%$ acreditavam que a HAS era uma doença grave e $70 \%$ deles admitiram utilizar farmacoterapia para controle pressórico.

A prática de atividade física era adotada por $30 \%$ dos indivíduos e, desse total, $60 \%$ se julgavam acima do peso, porém apenas $50 \%$ faziam dieta alimentar.

Da amostra, 20\% afirmaram ingerir bebida alcoólica socialmente, $80 \%$ negaram seu uso e $10 \%$ declararam ser tabagistas, com consumo médio de um maço por dia.

Dos entrevistados, 90\% negaram ser acometidos por DM e 10\% não sabiam informar; $40 \%$ mencionaram colesterol alterado, entretanto $40 \%$ não sabiam informar. 
A análise da Figura 1 que desenha globalmente o perfil do grupo Júpiter evidencia um padrão linear, cujas medidas pressóricas mantêm-se constantes. Essa tendência justifica-se principalmente pela compensação que os indivíduos exercem uns sobre os outros, ajustando, pois, os valores pressóricos para equilibrar seus níveis. Nesse caso, a amostra individual não afetou a coletiva, ainda que apresentasse um padrão irregular em alguns casos.

A Figura 2, que representa o indivíduo Netuno, apresenta variações nas medições para PAS, porém ocorre um padrão homogêneo para a PAD. Esse indivíduo tem história familiar pregressa, utiliza farmacoterapia contínua e é tabagista. Demonstrou certa resistência à

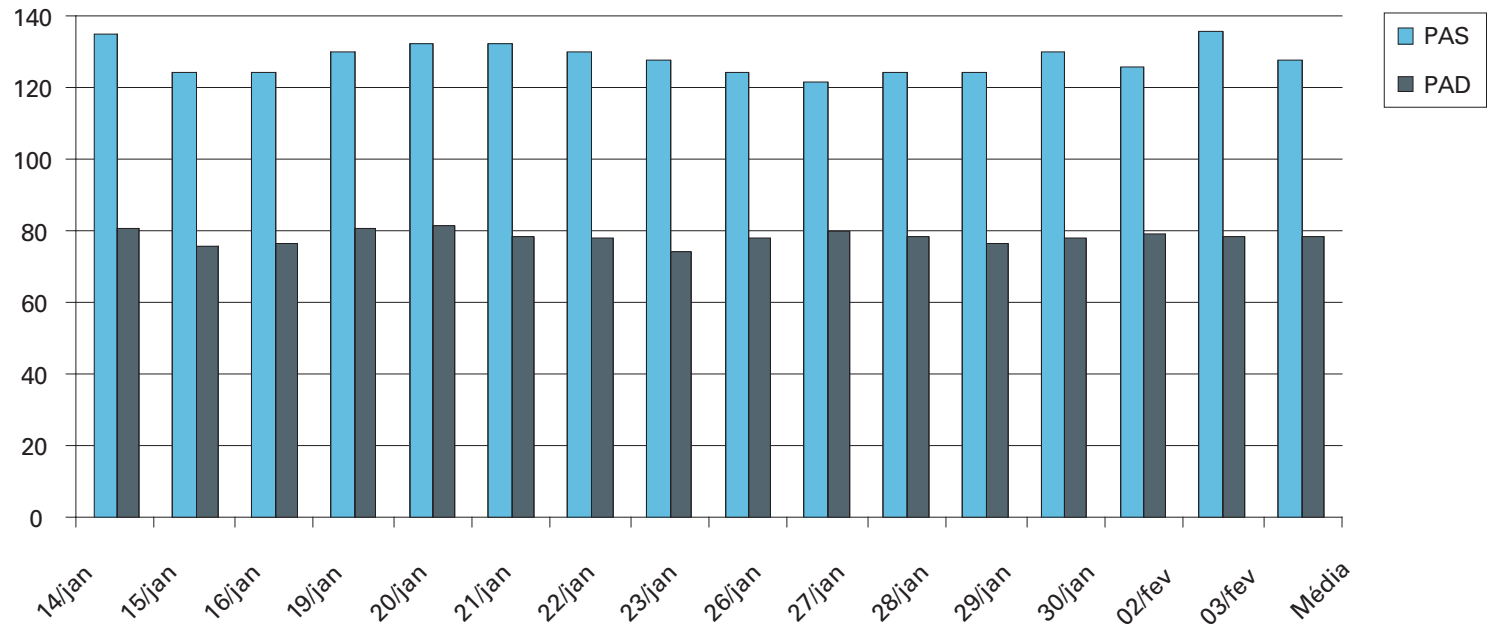

Figura 1: Evolução da pressão arterial - Grupo Júpiter

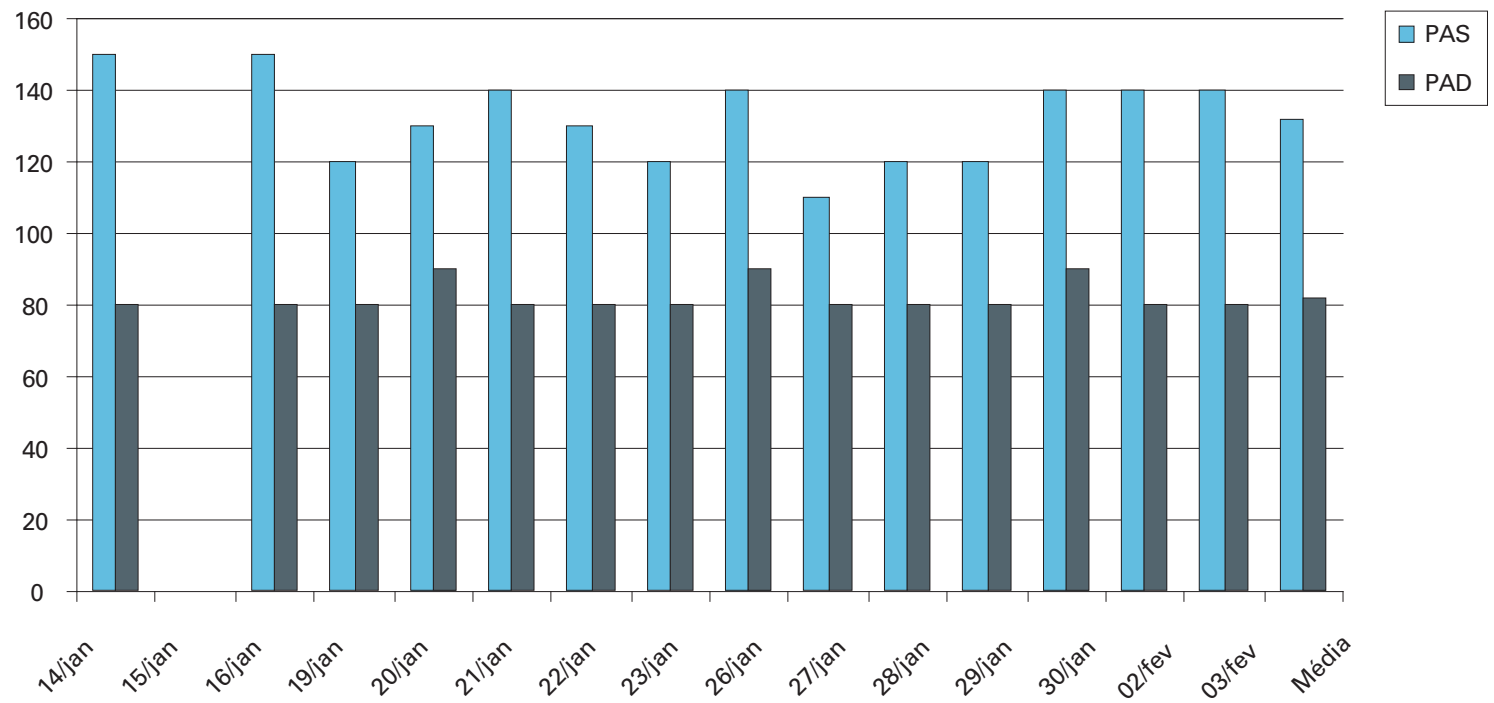

Figura 2: Evolução da pressão arterial - Netuno 
adesão ao protocolo, ainda que compreendesse sua importância.

O indivíduo Juno, representado pela Figura 3, apresenta um padrão irregular para as medições da PA. Esse indivíduo demonstrou grande compromisso com a pesquisa, porém dada uma limitação física não conseguiu aplicar o protocolo integralmente.
Os valores - registrados na Figura 4 - do indivíduo Plutão, ainda que tenha comparecido a apenas $73 \%$ das medições, demonstram bom controle pressórico. Neste caso, houve uma grande positividade em relação à pesquisa, adesão ao protocolo e satisfação com os resultados obtidos.

O indivíduo Minerva desenha um ótimo gráfico para validar o protocolo de intervenções

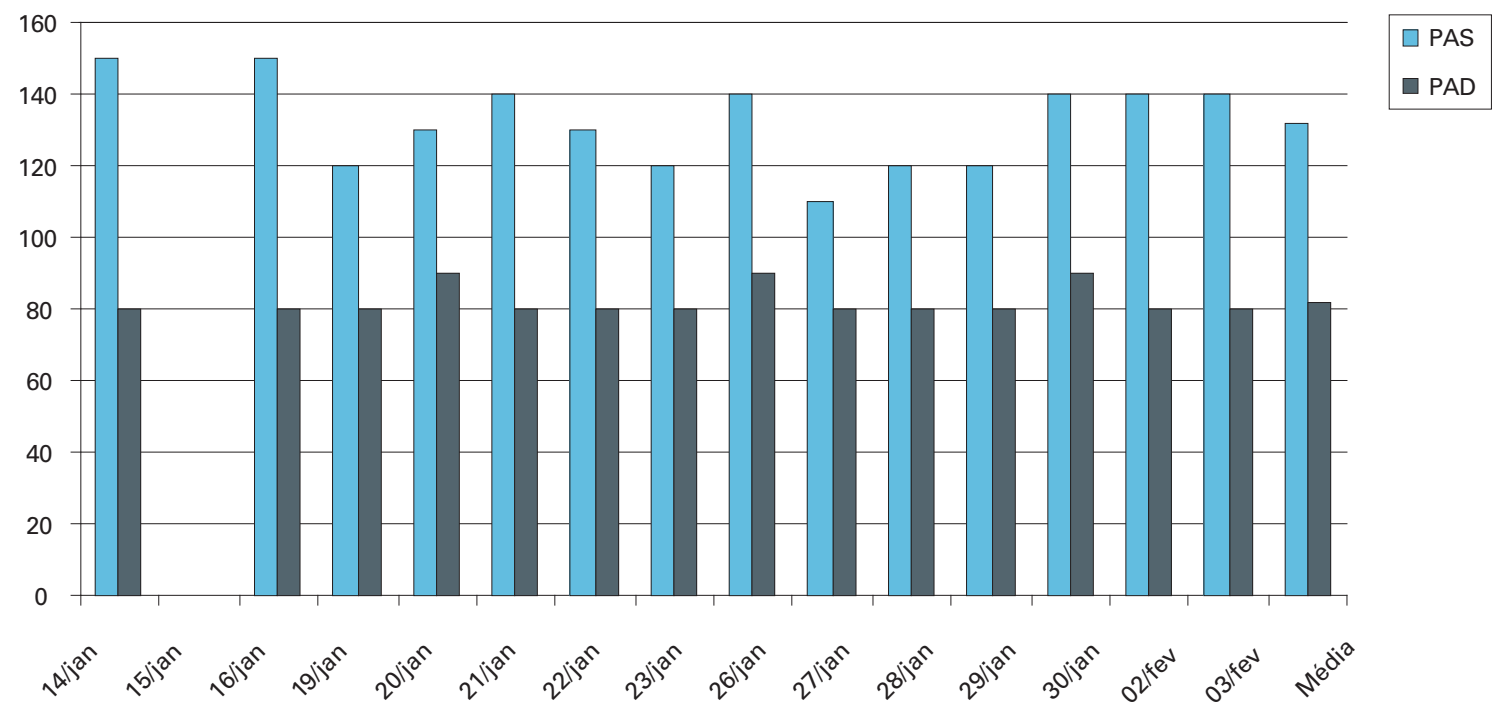

Figura 3: Evolução da pressão arterial - Juno

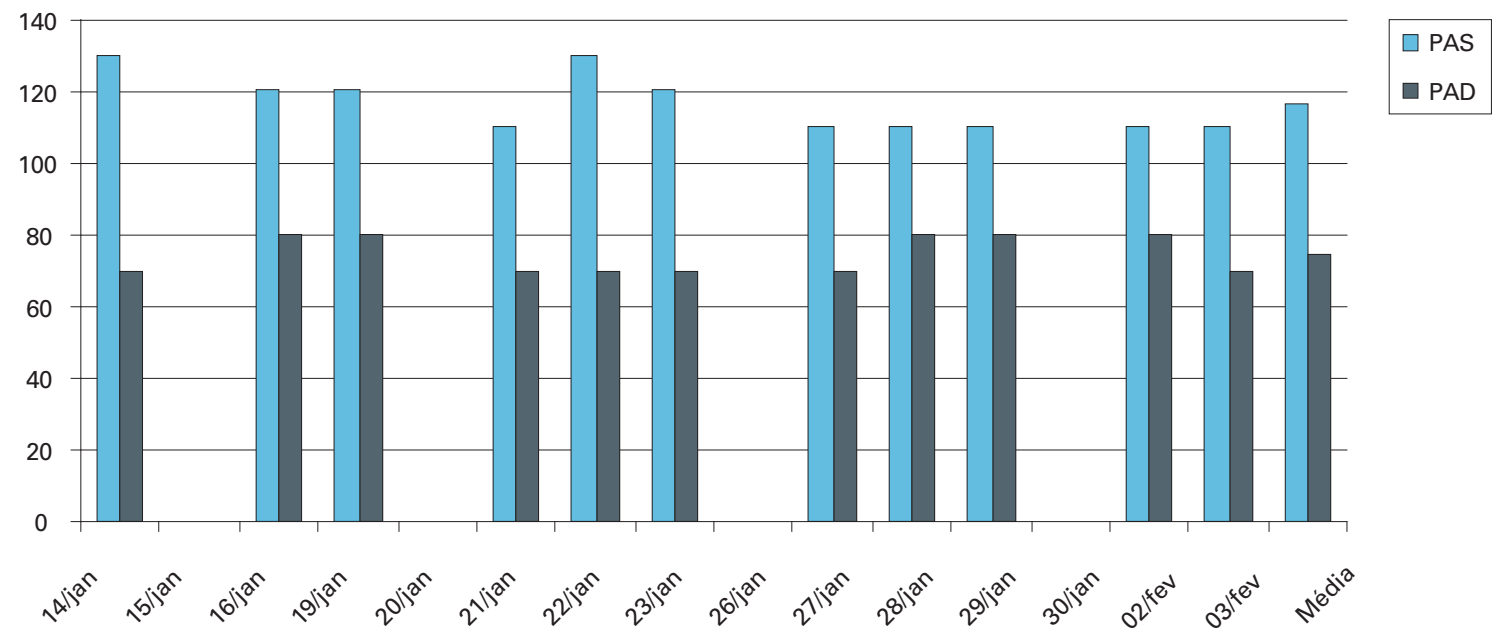

Figura 4: Evolução da pressão arterial - Plutão 
sugeridas na pesquisa. O comportamento pressórico evidencia, ao final das medições, uma queda de $20 \mathrm{~mm} / \mathrm{hg}$ para a PAS e demonstra um padrão irregular com oscilações não maiores que $90 \mathrm{~mm} / \mathrm{hg}$ para a PAD, conforme apresentado na Figura 5.

A evolução pressórica do indivíduo Febo demonstra ótimo controle, conforme apresentado na Figura 6. Esse indivíduo é um adulto jo- vem, com grande disposição para a adesão ao protocolo de condutas e faz uso de farmacoterapia contínua.

As medidas pressóricas registradas na Figura 7, do indivíduo Diana, apresentam picos para níveis da PAS coincidentemente associados a atritos familiares relatados pelo colaborador. O comportamento para PAD segue um padrão homogêneo. Da amostra total, este in-

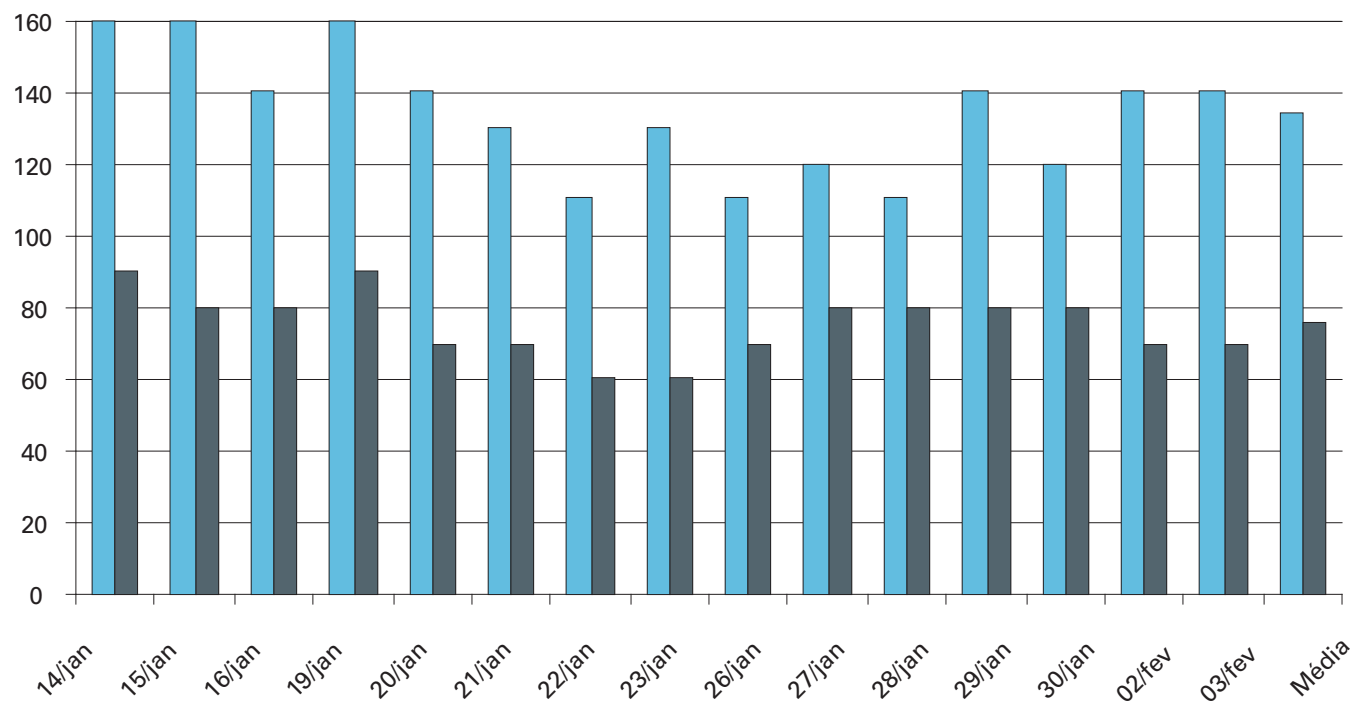

Figura 5: Evolução da pressão arterial - Minerva

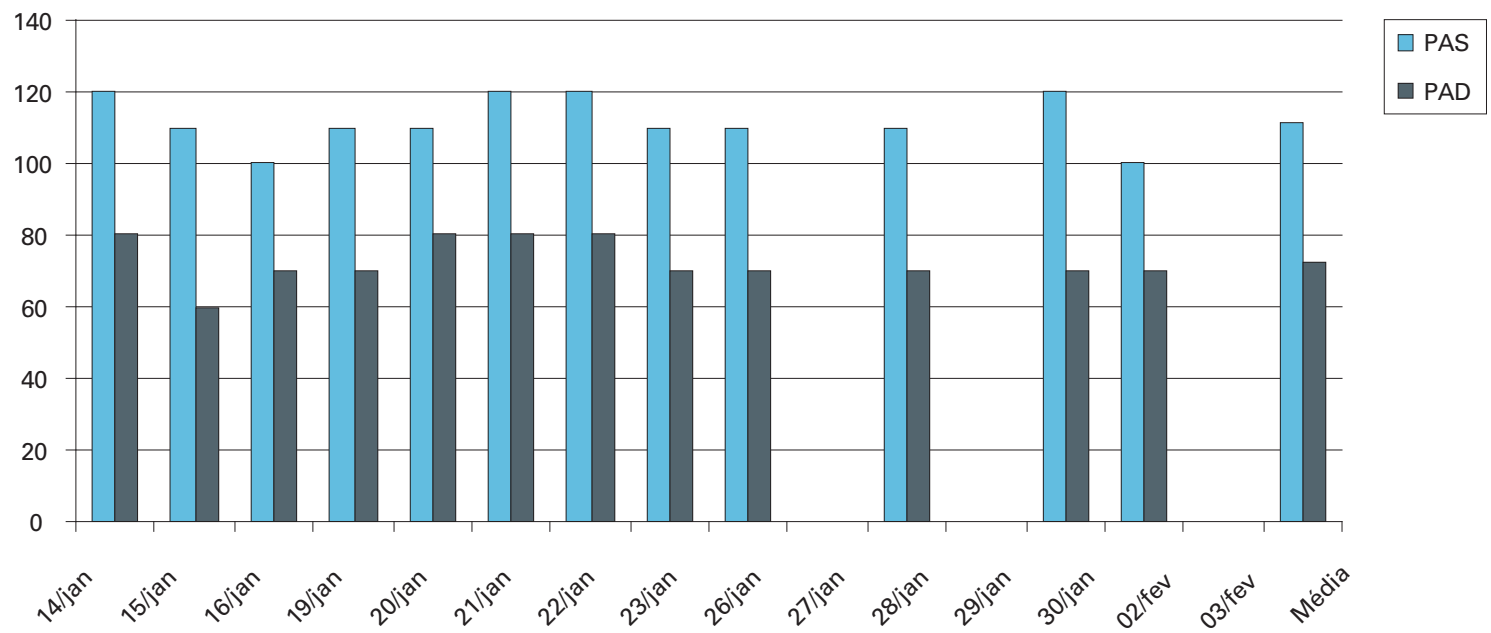

Figura 6: Evolução da pressão arterial - Febo 
divíduo representa o único que não acredita na gravidade da HAS e mostra grande tendência ao ceticismo religioso.

A Figura 8, representada pelo indivíduo Vênus, demonstra um padrão homogêneo tanto para a PAS quanto para a PAD. Um fator relevante a ser considerado é a questão do sobrepeso e histórico familiar positivo. Esse indivíduo apresentou parcial adesão ao protocolo sugerido.

O comportamento pressórico registrado nas Figuras 9, 10 e 11, respectivamente dos indivíduos Mercúrio, Marte e Vulcano, apresenta variações similares tanto em relação à PAS

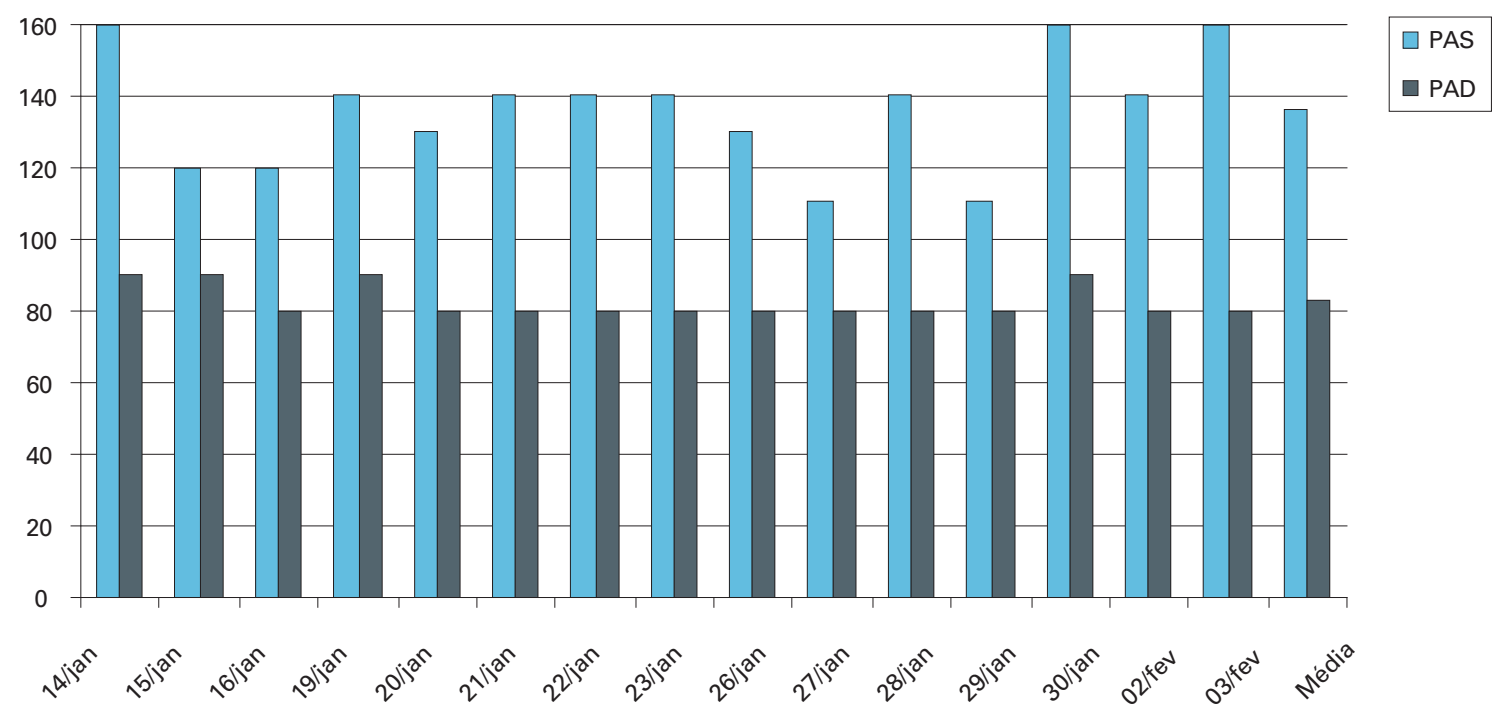

Figura 7: Evolução da pressão arterial - Diana

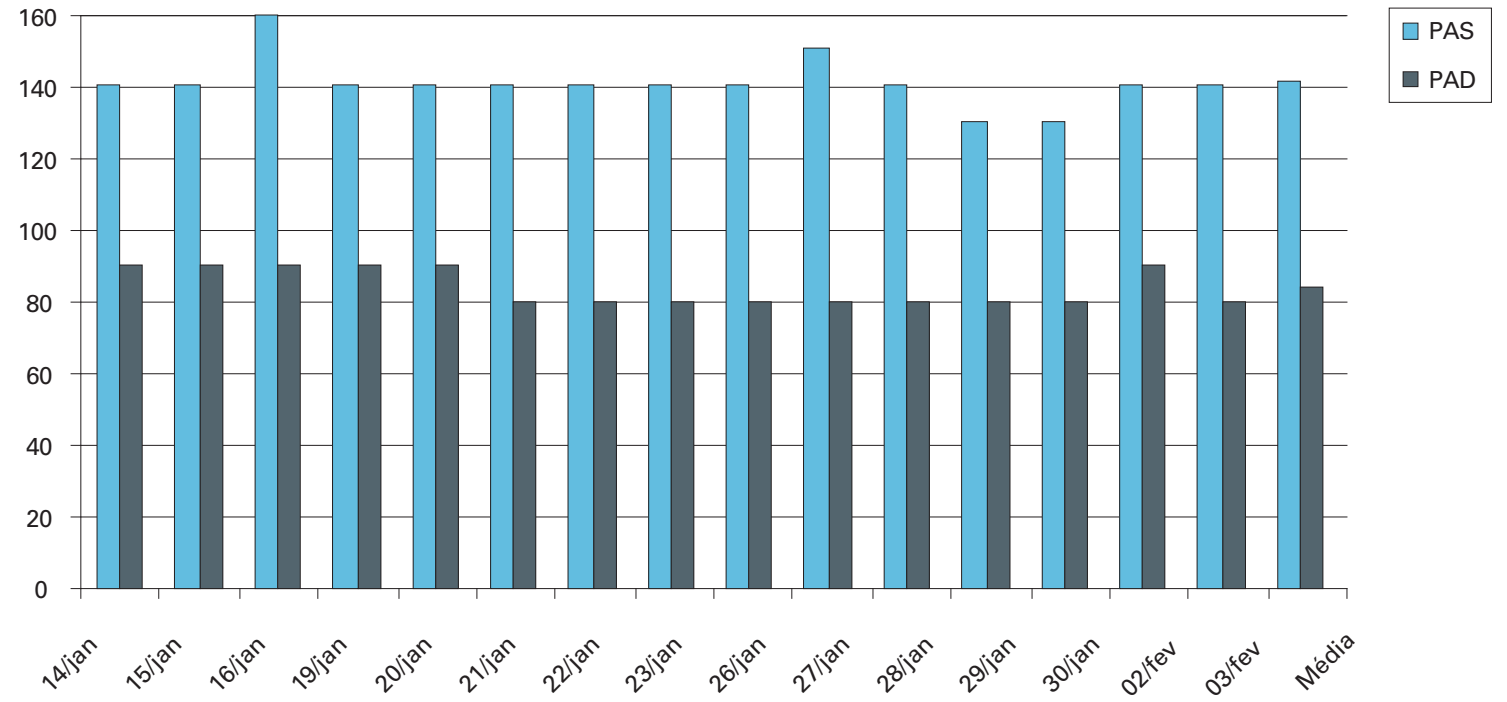

Figura 8: Evolução pressão arterial - Vênus 


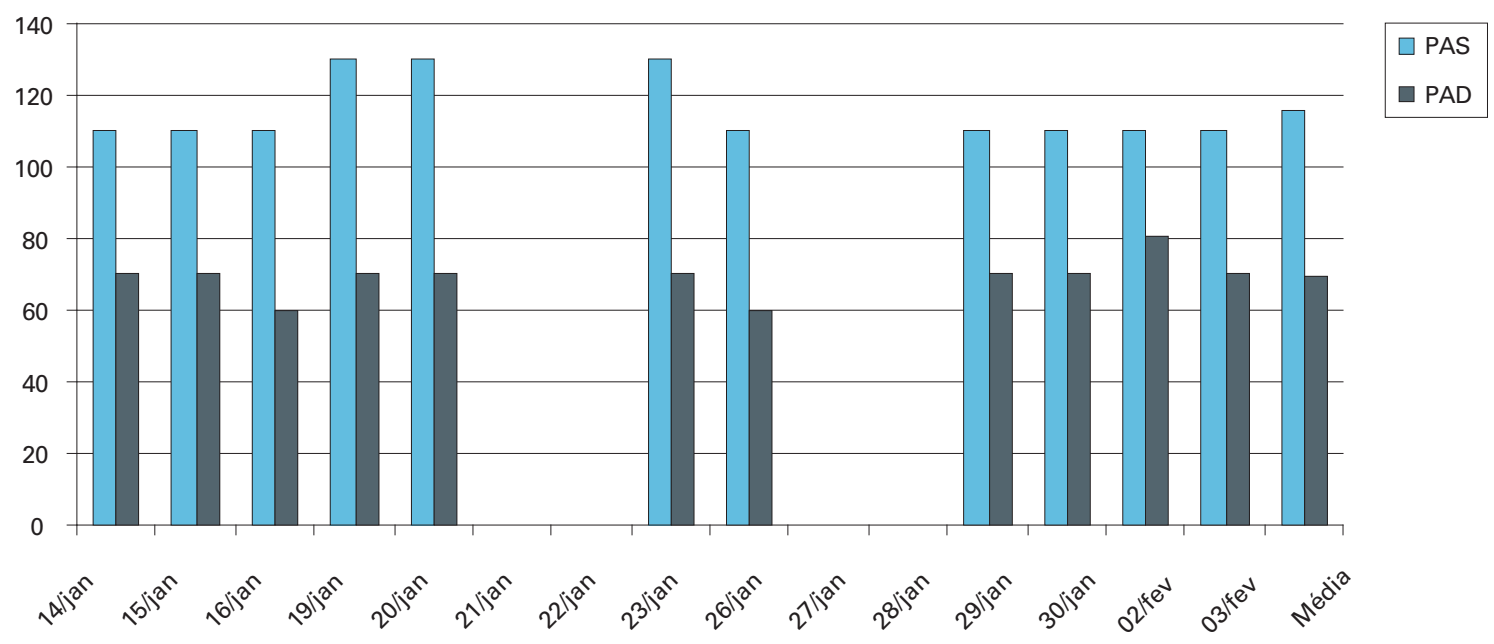

Figura 9: Evolução da pressão arterial - Mercúrio

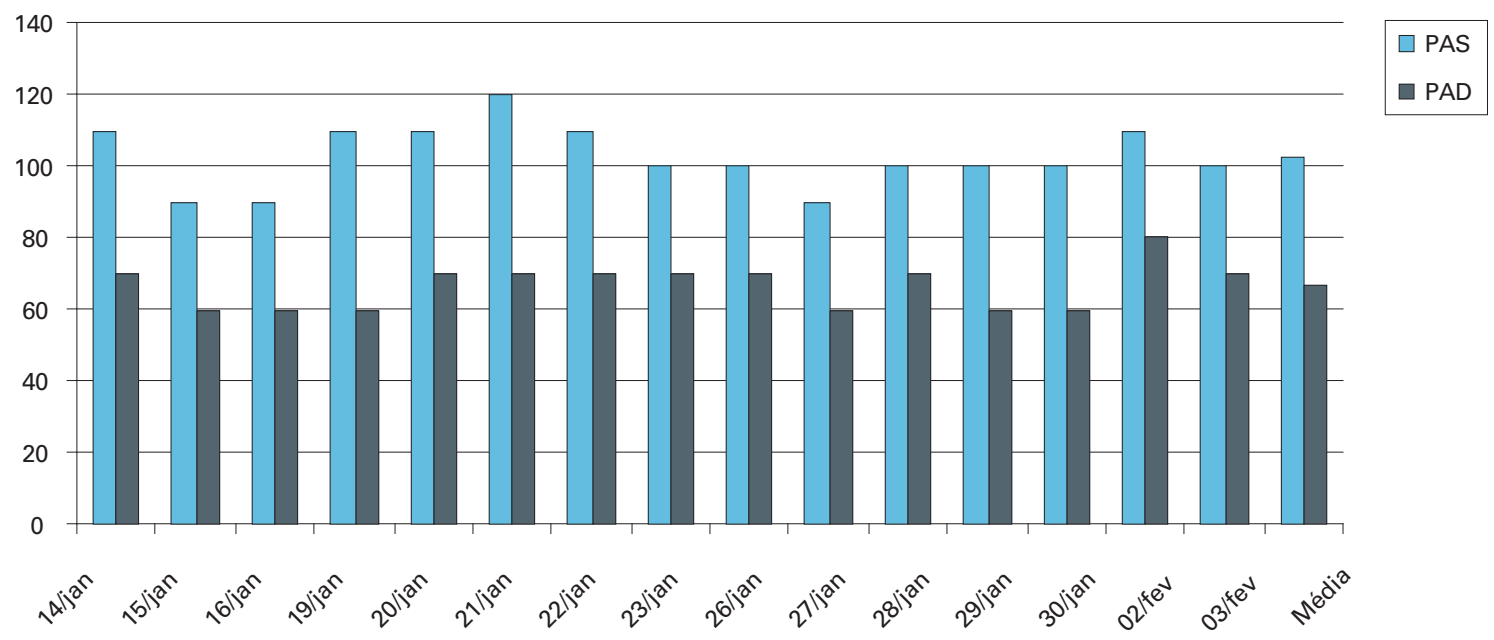

Figura 10: Evolução da pressão arterial - Marte

quanto à PAD, porém esses valores não comprometem os parâmetros pressóricos normais.

\section{Discussão}

A educação em saúde tem contribuído significativamente tanto para a prevenção quanto para o controle de doenças nos últimos 20 anos, principalmente quando se relaciona com os custos para a saúde, que podem ser redu- zidos por meio dessa estratégia. Sua proposta é fornecer conhecimento com a finalidade de estimular pacientes para efetivar mudanças em seu comportamento ${ }^{12}$.

Mudar o estilo de vida é uma tarefa difícil e quase sempre acompanhada de muita resistência; por isso, a maioria das pessoas não consegue fazer modificações e, especialmente, mantêlas por muito tempo. No entanto, a educação em saúde é uma alternativa fundamental para conduzir as pessoas a essas mudanças, para fins de 


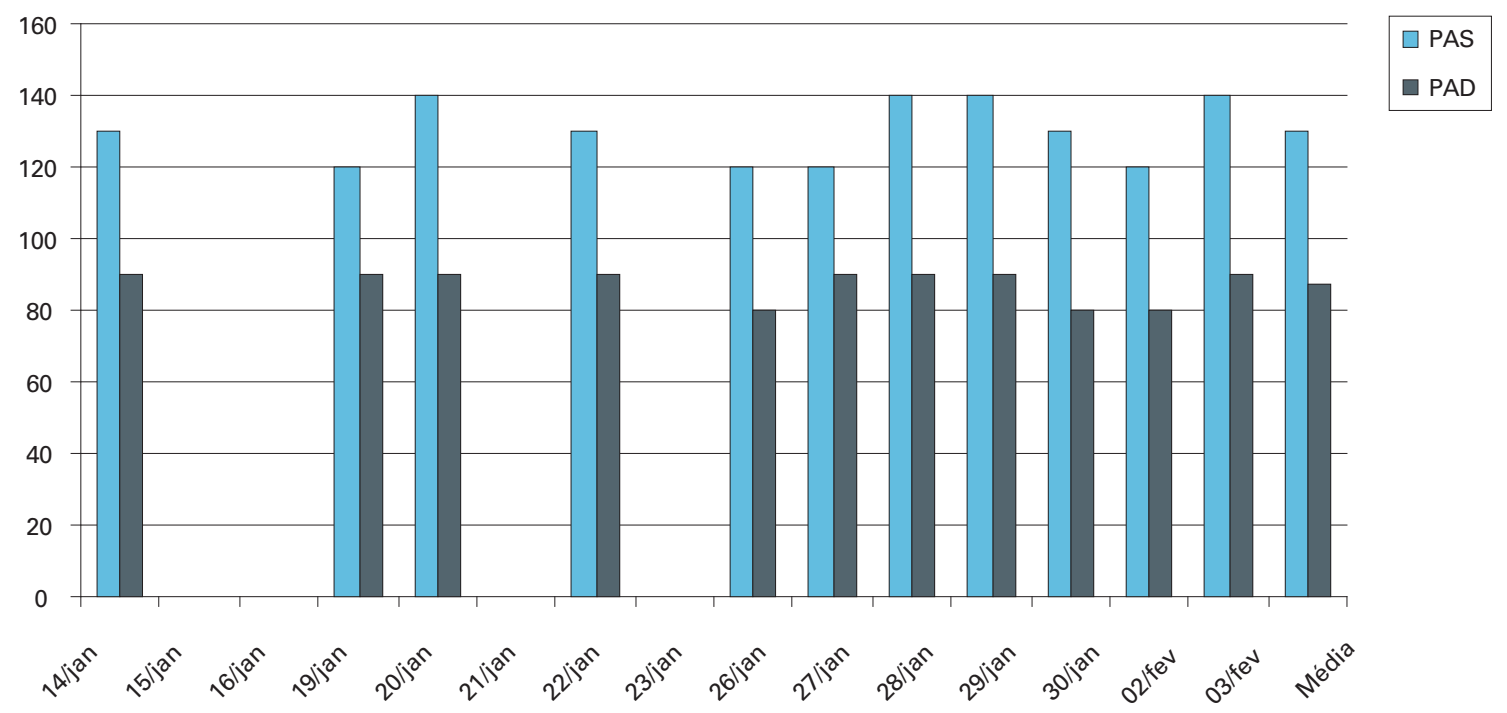

Figura 11: Evolução da pressão arterial - Vulcano

prevenção e/ou controle dos fatores de risco da HAS, por meio do cultivo de hábitos e atitudes saudáveis ${ }^{13}$.

O estudo desenvolvido propôs uma abordagem educativa ao paciente hipertenso para torná-lo mais cuidadoso em relação a sua saúde e mais preparado para o enfrentamento da doença.

Por ser a hipertensão arterial uma doença multifatorial, o desenvolvimento e a implementação de estratégias de intervenção, em particular a de educação em saúde, envolvem uma visão ampla do indivíduo, em que devem ser considerados aspectos tanto individuais quanto coletivos $^{12}$.

A análise estatística bruta fundamentada na variável PA, neste estudo, não é absoluta para identificar um padrão exato nas variações pressóricas, pois elas são ajustáveis a " $\mathrm{n}$ " variáveis, e não apenas à PA, o que permite considerar que o estudo abre uma abordagem ampla sobre as características comportamentais que lhe são aplicáveis.

Este estudo teve essencialmente uma abordagem estatística, estimulado pelas orientações do protocolo sugerido para medidas de controle da HAS, mas percebeu-se a necessidade de melhor compreensão de todos os processos que en- volviam o vínculo estabelecido com os colaboradores para um entendimento adequado.

Os sujeitos da pesquisa, com média de idade de 56 anos, constituem-se de pessoas simples, em que é notório um nível sociocultural baixo. Para adequar o protocolo de condutas a linguagem da amostra, foi elaborado um folder explicativo em que constavam todas as informações da intervenção sugerida, de forma clara e objetiva. No entanto, algumas barreiras de comunicação foram encontradas. Este fato foi constatado pelos seguintes comentários:

"Não sei lê quando chegar em casa meu filho lê pra mim" (DIANA).

“A letra é miudinha" (MERCÚRIO).

A amostra pesquisada demonstrou necessidade das medidas pressóricas diárias, não apenas intencionando fazê-los participar da pesquisa, mas também pelo fato de estarem sendo tratados de maneira especial, dando-lhes a devida atenção e procurando ouvi-los nas suas queixas e desejos. Os depoimentos denotam esses fatos: 
“Eu chego cedo não me importo o posto tá melhorando agora" (JUNO).

"Que bom que agora posso ficar vindo todo dia e encontrar vocês" (MINERVA).

A adesão ao protocolo sofreu certa resistência de alguns sujeitos. A diminuição do sal na dieta foi um dos itens com maior rejeição, seguida pelo decréscimo do tabaco. As atividades físicas sugeridas ganharam grande positividade em relação à amostra, porém nem todos se prontificaram a adotá-la. Os respectivos depoimentos demonstram as questões pontuadas:

“Tirá o sal todo é muito ruim" (DIANA).

"Só um pouquinho de sal não tem problema" (NETUNO).

"Sem sal é difícil" (PLUTÃO).

“Eu já fumo pouco só um maço por semana" (NETUNO).

"Eu tô caminhando todo dia de manhã e a tarde" (DIANA).

“De dia eu cuido do meu netinho e a tarde caminho" (MINERVA).

“Não posso caminhar tenho perna mecânica" (JUNO).

Os níveis pressóricos na amostra estudada sofreram oscilações relevantes quando associadas a questões emocionais. Esse fato foi constatado com a seguinte afirmação:

"Minha neta saiu de casa brigou comigo foi morar com a mãe dela não me conformo ela não fala mais comigo já fico vermelha logo" (DIANA).

Alguns sujeitos da pesquisa questionaram a necessidade da continuidade da farmacoterapia, visto que os níveis pressóricos permaneciam con- trolados. No entanto, foram orientados sempre a seguir conduta médica. Veja este depoimento:

"A pressão tá normal acho que vou tomar só meio comprimido senão abaixa muito" (MARTE).

Em outra situação, um dos sujeitos da pesquisa, em consulta médica, mostrou o folder da pesquisa, com os valores pressóricos medidos, ao cardiologista que elogiou a iniciativa da pesquisa. Como relata a seguir o colaborador:

"Ele ficou feliz disse que não sabia que a Uninove fazia isso também” (PLUTÃO).

Foi elaborado um Certificado de Reconhecimento como forma de contemplar os colaboradores pela importante contribuição prestada à pesquisa. Esse simples gesto comoveu alguns colaboradores, como comprovam os seguintes relatos:

"O último diploma que eu ganhei foi em 1960, não acredito!" (PLUTÃO).

“Vou mostrar pro meu filho" (DIANA).

\section{Conclusão}

Concluímos que as estratégias para a prevenção da HAS são de grande importância, especialmente no âmbito da atenção primária, e produzem consideráveis impactos socioeconômicos. Deve-se ressaltar que o profissional enfermeiro, na prática de suas atribuições, deve assumir a postura de educador e zelar pela promoção da saúde e prevenção de agravos da comunidade com o fito de torná-la conhecedora dos processos saúde-doença para que tenha o entendimento necessário sobre a HAS e todos os contextos que lhe são pertinentes. O comparecimento assíduo de apenas $50 \%$ da amostra e o tempo de 15 dias adotado para desenvolvimento da pesquisa contribuíram diretamente para resultados limitados. Considera-se, entretanto, 
que o maior ganho não está analisado estatisticamente, tampouco figura em gráficos. Trata-se das relações humanas que se estabeleceram no desenvolvimento da pesquisa. O grupo mostrou motivação e colaboração durante todo o processo instituído e buscou entendimento sobre a HAS e as formas de combatê-la, de maneira prática e fácil. Ficaram evidentes a importância e o respeito que obtivemos em relação aos sujeitos da pesquisa.

Observamos ainda que a pesquisa favoreceu a aproximação entre a comunidade e a Unidade Básica de Saúde naquela região, dando credibilidade ao serviço e reconhecimento aos profissionais atuantes.

\section{Referências}

1. Boing AC, Boing AF. Hipertensão arterial sistêmica: o que nos dizem os sistemas brasileiros de cadastramentos e informações em saúde. Rev Brasil Hipert. 2007;14: 84-88.

2. Laterza $M C$, Rondon MUPB, Negrão CE. Efeito anti-hipertensivo do exercício. Rev Bras Hipert.abr./ jun.2007;14(2):104-111.

3. Santoro DC, Stipp MAC, Leite JL, Stipp CA. O cliente com doença coronariana e os fatores de risco In: Figueiredo NMA, Stipp MAC, Leite J L editores. Cardiopatias avaliação e intervenção em enfermagem. São Paulo: Yendis;2006:81-101.

4. Santos ZMSA, Lima HP. Tecnologia educativa em saúde na prevenção da hipertensão arterial em trabalhadores: análise das mudanças no estilo de vida. Texto contexto - enferm. [Acesso em 14 set. 2008];17(1):90-97.Disponível em: http://www. scielo.br/scielo. php?script=sci_arttext\&pid=S010407072008000100010\&lng=pt\&nrm=iso. doi: 10.1590/ S0104-07072008000100010.

5. Nunes APOB, Rios ACS, Cunha GAB, Pereira AC, Negrão CE. Efeitos de um programa de exercício físico não supervisionado e acompanhado a distância, via internet, sobre a pressão arterial e composição corporal em indivíduos normotensos e pré-hipertensos. Arq Bras Cardiol. 2006 abr. [Acesso em 14 set 2008];86(4):289-296. Disponível em: http://www.scielo.br/scielo.php?script=sci_ arttext\&pid=S0066-782X2006000400009\&lng=pt\&nr $\mathrm{m}=$ iso. doi: $10.1590 /$ S0066-782X2006000400009.
6. Campana EMG, Brandão AA, Pozzan RM, Campos ME, Brandão AP. Hipertensão Arterial Sistêmica. Jorn Brasil Medic. 2007 mar.;92(3):55-64.

7 Silva MEDC, Barbosa LDCS, Oliveira ADS, Gouveia MTO, Nunes BMVT, Alves ELM. As representações sociais de mulheres portadoras de Hipertensão Arterial. Rev Bras Enferm. 2008 ago. [Acesso em 14 set. 2008];61(4): 500-507. Disponível em: http://www. scielo.br/scielo.php?script=sci_arttext\&pid=S003471672008000400017\&lng=pt\&nrm=iso.

8. Baldissera VDA, Carvalho MDAB. Queixas mais comuns no Ambulatório de controle da hipertensão Arterial. Arquivos de Ciências da Saúde da UNIPAR maio/ago. 2004;8:101-106.

9. Rolim MO, Castro ME. Adesão às orientações fornecidas no Programa de Controle da Hipertensão: uma aproximação aos resultados padronizados de enfermagem. On-line Brazilian Journal of Nursing. 2007;6(1).

10. Jardim PCBV, Gondim MRP, Monego ET, Moreira HG, Vitorino PVO, Souza WKSB et al. Hipertensão arterial e alguns fatores de risco em uma capital brasileira. Arq Bras Cardiol. Abr. 2007[Acesso em 14 Set 2008];88(4):452-457. Disponível em: http://www. scielo.br/scielo.php?script $=$ sci_arttext\&pid $=$ S0066782X2007000400015\&lng=pt\&nrm=iso. doi: $10.1590 /$ S0066-782X2007000400015.

11. Pickering TG, Hall JE, Appel LJ. Recommendations for blood pressure measurement in humans and experimental animals. Hypertension. 2005;45: 142-61.

12. Chaves ES, Leite LIM, Araújo TL, Damasceno MMC. Eficácia de programas de educação para adultos portadores de hipertensão arterial. Rev Bras Enferm. [serial on the Internet]. 2006 Aug. [acesso 17 mar. 2009];59(4): 543-547. Disponível em: http://www. scielo.br/scielo.php?script=sci_arttext\&pid=S003471672006000400013\&lng=en. doi: 10.1590/S003471672006000400013 .

13. Santos ZMSA, Lima HP. Tecnologia educativa em saúde na prevenção da hipertensão arterial em trabalhadores: análise das mudanças no estilo de vida. Texto contexto - enferm. [serial on the Internet]. Mar. 2008[acesso em 17 mar. 2009];17(1):90-97. Disponível em: http://www. scielo.br/scielo.php?script=sci_arttext\&pid=S010407072008000100010\&lng=en. doi: 10.1590/S010407072008000100010 . 
14. Araujo JC, Guimarães AC. Controle da hipertensão arterial em uma unidade de saúde da família. Rev Saúde Pub. 2007 [acesso em 15 ago.2008].41(3):368374. Disponível em: http://www.scielo.br/ scielo.php?script=sci_arttext\&pid=S003489102007000300007\&lng=. doi: 10.1590/S003489102007000300007.
15. Massironi MMG. Protocolo de tratamento da hipertensão arterial e da diabetes melito tipo 2 na atenção básica. Secretaria Municipal da Saúde. São Paulo;2008:15-17. 